\section{Practising evidence based medicine}

Both developed and developing countries of the world, enjoy accomplishments of public health. An example from Sri Lanka is the gain in life expectancy during the twentieth century where much of this increase can be attributed to population based public health interventions such as control of malaria, reduction of maternal deaths and provision of safe water and food.

Despite these successes, many other public health challenges remain and new challenges emerge.

To address these challenges in public health and to meet the expectations of continuing improvement, a drive towards a more widespread use of evidence-based strategies has been recommended (1).

Ideally, public health practitioners should always incorporate scientific evidence in developing policies, making management decisions and in implementing interventions. In reality however, these decisions are often shown to be based on short term demands rather than on long term plans: and policies and programmes are often developed around anecdotal evidence. It has been stated that decision making in public health is often driven by "crises, hot issues, and concerns of organised interest groups" (1).

To address these issues in a more evidence based approach does not require extensive formal training in epidemiology, biostatistics or behavioural sciences but only requires enhancing individual skills for wider use of data and available analytical tools. Data on the importance of a particular health problem and its link with some preventable risk factors would constitute evidence that 'something should be done' and data on relative effectiveness of alternative interventions would assist in selecting 'what exactly should be done'.

We, public health practitioners can be categorised into several user groups who should use of evidence for various public health decision making.

Public health practitioners who are the policy makers at local, regional, national and international levels are faced with macro level decisions on how to allocate the public resources depending on the importance of a particular health problem and its preventability. This group has the additional responsibility of creating a more favourable organisational climate for practising evidence based public health by convincing politicians of 'what exactly should be done' based on available evidence.

Public health practitioners with responsibilities for strategic planning would need evidence on the relative effectiveness of alternative interventions to address health problems.

However, more often than not public health practitioners have only a narrow range of options if funds are earmarked for a specific purpose. Still practitioners have the opportunity, even the obligation, to survey the evidence carefully for alternative ways to achieve the desired goals. They have the responsibility of seeking evidence on how they can be most effective and efficient in achieving these goals.

Public health practitioners sometimes belong to key stakeholder groups when interest groups are formed to support or oppose specific policies dealing with a problem, such as legality of abortions. While ideology fuels passion on these issues, the use of evidence can temper views or suggest a feasible range of solutions for compromise among opposing views.

Public health practitioners who are researchers on population health issues, who evaluate the impact of specific policies or programmes, both collect and use evidence to explore preventability of a problem and effectiveness of alternative strategies.

Whichever category we belong to, if we, public health practitioners, practise evidence based strategies in our work, a higher health return on the tax payers investments could be achieved, and public health practitioners would be more successful in competing for limited resources as they have evidence to support their action and justify the investment.

1. Brownson RC, Baker EA, Leet TL, Gillespie $\mathrm{KN}$. Evidence-based public health, Oxford: Oxford University Press, 2003.

\section{Nalika Gunwardena}

